\title{
A Red Phosphorus-Graphite Anode for K-ion Batteries
}

\author{
Isaac Capone ${ }^{\mathrm{a}}$, Jack Aspinall ${ }^{\mathrm{a}}$, Hyeon Jeong Lee ${ }^{\mathrm{a}}$, Albert W. Xiao ${ }^{\mathrm{a}}$, Johannes Ihli ${ }^{\mathrm{a}}{ }^{\mathrm{b}}$, and \\ Mauro Pasta ${ }^{a},{ }^{*}$

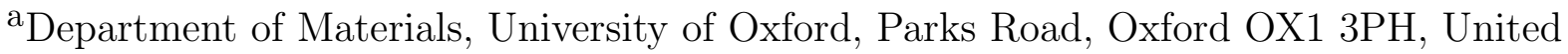 \\ Kingdom \\ ${ }^{b}$ Paul Scherrer Institute, Forschungsstrasse 111, 5232 Villigen PSI, Switzerland \\ *Corresponding author: mauro.pasta@ox.ac.uk
}

\begin{abstract}
Red phosphorus (RP) is a promising anode material for potassium-ion batteries because of its theoretical capacity of $865 \mathrm{~mA} \mathrm{~h} \mathrm{~g}^{-1}$ delivered at an average potential of $0.5 \mathrm{~V} \mathrm{vs} \mathrm{K}^{+} / \mathrm{K}$. However, its alloy reaction to form KP entails a volume expansion of $162 \%$ resulting in severe stresses that lead to SEI and electrode fracture, loss of electric contact, and ultimately reduced cycle life. Moreover, its low electronic conductivity $\left(10^{-14} \mathrm{~S} \mathrm{~cm}^{-1}\right)$ limits rate capability. Here, we report a RP-graphite composite prepared by a two step ball milling procedure to control particle size and optimize carbon coating. Electrodes prepared with the composites achieve high capacity $\left(723 \mathrm{~mA} \mathrm{~h} \mathrm{~g}_{\mathrm{P}}^{-1}\right)$ at $\mathrm{C} / 20$ and retaining $75 \%$ at $5 \mathrm{C}$. It also shows very good cycling stability, retaining more than $96 \%$ of the capacity after 100 cycles at $1 \mathrm{C}$.
\end{abstract}

The demand for lithium-ion batteries (LIBs) is expected to increase tenfold by 2030 due to a widespread uptake of electric vehicles $(\mathrm{EVs}) .1,2$ Concerns have been raised about the availability and cost of lithium in the near-future, because of its geographical concentration. 3 3 More abundant alkali metals such as sodium and potassium are being considered, due to their higher abundance and lower cost than $\mathrm{Li}$ (47 billion tons in the largest deposit available, 7 billion tons and 80 million tons, for soda ash, potash and Li precursors, respectively, resulting in an average cost of 200,1000 , and 8,750 USD tons $^{-1}$ ). For the successful uptake of alternative chemistries in most transport applications, they must at least match the energy density of commercial LIBs. 3 [4, 5. State of the art commercial Li-ion batteries employ a silicon oxide doped graphite anode and a nickel-manganese-cobalt oxide cathode, storing $250 \mathrm{Wh} \mathrm{kg}^{-}$1. 6. 7. To extend the electrification of transport to aviation, heavy goods vehicles and shipping, higher energy densities of about $500 \mathrm{Wh}$ $\mathrm{kg}^{-} 1$ are required. 8

Potassium-ion batteries (KIBs) are a promising alternative to LIBs as $\mathrm{K}^{+}$exhibits a low reduction potential similar to that of $\mathrm{Li}(-2.93 \mathrm{~V}$ vs SHE for $\mathrm{K},-3.01 \mathrm{~V}$ vs $\mathrm{SHE}$ for $\mathrm{Li}$ ). However, the larger size of the ion compared to $\mathrm{Li}^{+}(1.38 \AA$ vs 0.76 $\AA)$ introduces new challenges into the research of suitable electrode materials. Traditional transition metal oxides cathodes, for example, are structurally unstable whilst electrochemically cycling with $\mathrm{K}^{+}$ ions. 9 [1] Notably prussian blue analogues have shown good performance due to their ability to store $\mathrm{K}^{+}$ions with minimal structural change. 12 Like $\mathrm{Li}, \mathrm{K}$ is able to intercalate into graphite forming the phase $\mathrm{KC}_{8}$, with a theoretical capacity of $279 \mathrm{mAhg}^{-1}$ at a very low potential $(0.25 \mathrm{~V}$ vs $\left.\mathrm{K}^{+} / \mathrm{K}\right) \cdot 13$, 14. Graphite is an appealing negative electrode thanks to its low cost and ease of manufacture, but unfortunately, the energy density of the intercalation of $\mathrm{K}^{+}$into graphite is lower than that of $\mathrm{Li}^{+}\left(279\right.$ vs $\left.376 \mathrm{mAhg}^{-1}\right)$. The development of a potassium anode with high energy density is a crucial step for the energy density of KIBs to be competitive with current and future LIBs.

Red phosphorus (RP) recently earned attention 


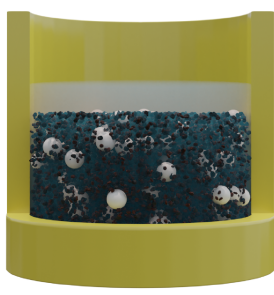

(1)

Red phosphorus wet milling $(\mathrm{d} 90<0.79 \mu \mathrm{m})$

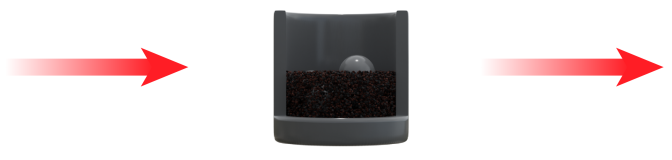

(2)

RP-graphite composite dry milling

(7:3 mass ratio)

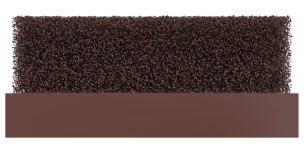

(3)

\section{Electrode fabrication}

Figure 1. Schematic of the production process of the electrode material. 1) RP is wet milled in ethylene glycol to reduce its particle-size distribution. 2) The ball milled RP is mixed with graphite using a 7:3 mass ratio via dry milling. 3) The resulting composite is mixed with Super P and the binder to fabricate the electrode. Experimental details are illustrated in the experimental section.

due to its ability to alloy with potassium (KP) to deliver a very high theoretical capacity of $865 \mathrm{~mA} \mathrm{~h} \mathrm{~g}_{\mathrm{P}}^{-1} \cdot 15,16$ The drawbacks of phosphorous are a very low electronic conductivity $\left(10^{-14} \mathrm{~S} \mathrm{~cm}^{-1}\right)$ and a large volume expansion (162\%) during the alloying reaction. Although it has been recently demonstrated that the stresses developed as a consequence of the volume expansion do not cause the fracture and failure of the particles - as occurs in crystalline $\mathrm{Si}$ with $\mathrm{Li}$ - the expansion/contraction process can cause the formation of cracks on the electrode material, followed by delamination and loss of electric contact, reducing its capacity over successive cycles. 17, 18 The preparation of RP-carbon composites has been demonstrated to be an effective way to mitigate these two drawbacks. In particular, ball milling has been proposed as a cheap, easy and scalable method to prepare these composites. 15, 16, 19 In a previous work, we demonstrated that the reduction of the particle size of phosphorus is an effective way to mitigate the stresses on the material and improve its cycle life with $\mathrm{Na} .20$ In the present work, we show the performance of that material with K. 20]. We prepared a RP-graphite composite where the RP particle size distribution was first reduced by ball milling, then mixed with graphite via another ball milling step to obtain the composite (Figure 1). This material demonstrated cycling performance with $\mathrm{K}$ superior to previous works, notably delivering a high first charge capacity $\left(723 \mathrm{~mA} \mathrm{~h} \mathrm{~g} \mathrm{~g}_{\mathrm{P}}^{-1}\right)$ and retaining most of its capacity even at high current rates such as $5 \mathrm{C}$.

To characterize the electrochemical performance of the RP-graphite composite, galvanostatic cycling experiments were performed. The material was tested in a three electrodes setup in a Biologic VMP3, using a EL-cell with $\mathrm{K}$ metal as both the counter and the reference electrode. The experiments were done in triplicate. Potassium bis(fluorosulfonyl)imide (KFSI) 1M in EC:DEC 1:1 was used as the electrolyte. The electrodes were cycled in the potential range $0.01-2.5 \mathrm{~V}$ vs $\mathrm{K}^{+} / \mathrm{K}$ at $\mathrm{C}$ rates ranging from $\mathrm{C} / 20$ to $5 \mathrm{C}$. In this work, we use "discharge" and "charge" to refer to the "potassiation" and "depotassiation" processes, respectively. For the operando Raman measurement, a 2032 coin cell was adapted in order to be used as operando cell. A $4 \mathrm{~mm} \varnothing$ hole was drilled through the center of the positive side of the cell and a $7 \mathrm{~mm}$ $\varnothing$ sapphire window was glued on the inside of the coin cell part using epoxy, to allow the investigation of the electrode material from outside the cell whilst cycling. The cell was assembled using the standard procedure. In the operando experiments, a current rate of $\mathrm{C} / 20$ was applied. Impedance analysis was performed on a three electrode cell with frequencies ranging $200 \mathrm{kHz}-1 \mathrm{~Hz}$, allowing a $1 \mathrm{~h}$ rest after every discharge/charge step.

To study the electrochemical performance of this material, galvanostatic cycling at $\mathrm{C}$-rates ranging from $\mathrm{C} / 20$ to $5 \mathrm{C}$ was performed. In Figure 2 the electrochemical performances of the RP-graphite 

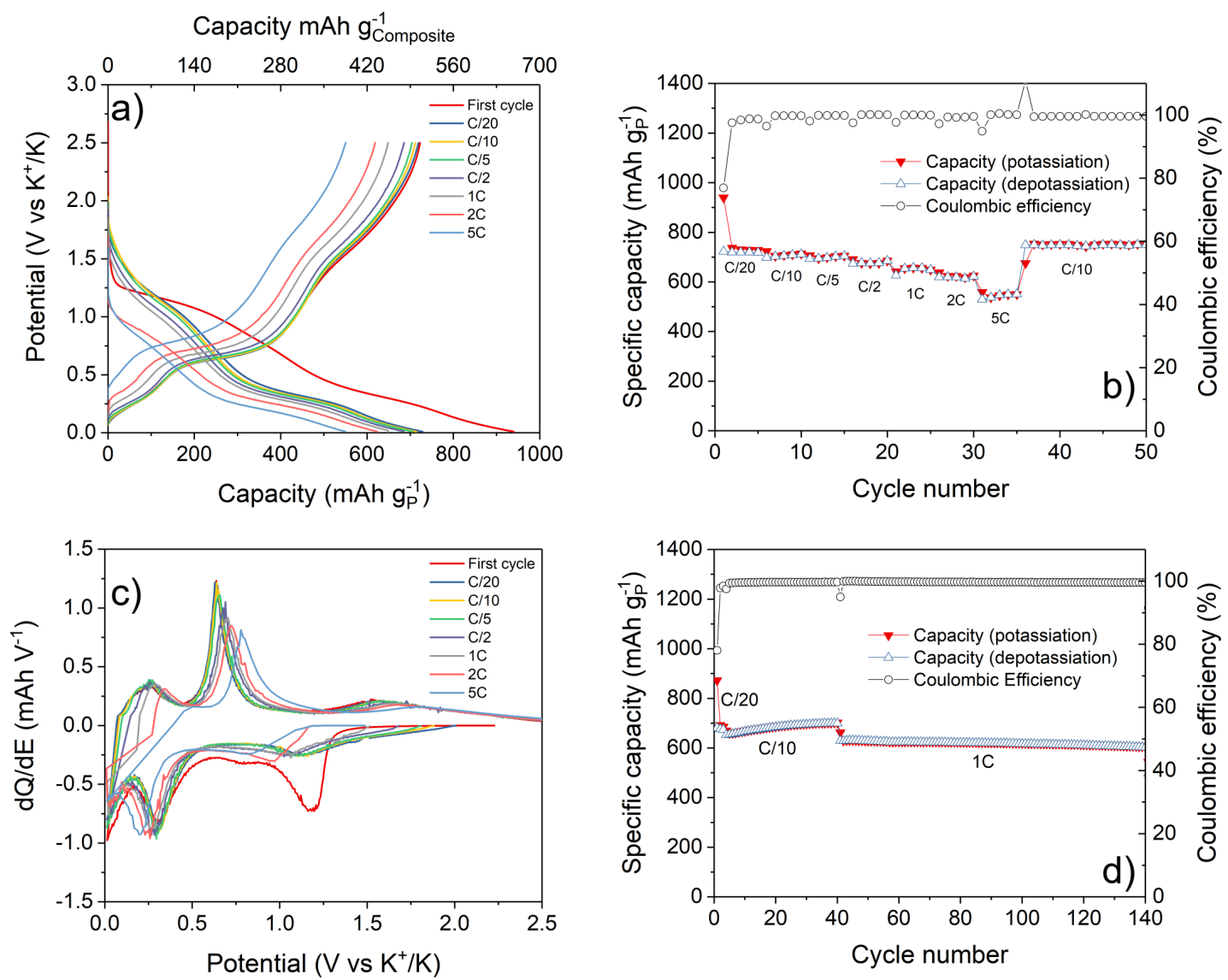

Figure 2. Electrochemical performance of RP-C composite. a) shows the galvanostatic profiles of the active material at increasing rates and (b) shows the rate capability of the material. In (c) the differential plots of the cycles shown in (a) are shown. d) Cycling stability of the RP-graphite composite at 1C.

composite are shown. On the first cycle, the galvanostatic profile shows two plateaus. The first one, centered at about $1.2 \mathrm{~V}$ vs $\mathrm{K}^{+} / \mathrm{K}$, is mainly related to the formation of the SEI. The second slope, occurring at about $0.3 \mathrm{~V}$ vs $\mathrm{K}^{+} / \mathrm{K}$, is likely related to the progressive formation of the KP alloy as the final product of this electrochemical reaction, as Yang et al. predicted (Figure 2a). 21] On the first cycle, the material delivered a discharge capacity of $939 \mathrm{mAhg}_{\mathrm{P}}^{-1}$ and an initial charge capacity (ICC) of $723 \mathrm{mAhg}_{\mathrm{P}}^{-1}$, with a coulombic efficiency of $77 \%$. The greater-than-theoretical capacity, as well as the low coulombic efficiency on the first discharge is mainly due to the formation of the SEI. The material was then tested at increasing C-rates up to $5 \mathrm{C}$ (Figure 2 $\mathrm{b}$ ), showing a good rate capability, delivering $86.3 \%$ of the ICC when cycled at $2 \mathrm{C}$ and $75.8 \%$ of the ICC when cycled at $5 \mathrm{C}$.
The differential capacity plot in Figure $2 \mathrm{p}$ shows peaks related to the formation of the alloy and intermediate phases during cycling. Only on the first cycle, we observe a peak in discharge at about $1.2 \mathrm{~V}$ vs $\mathrm{K}^{+} / \mathrm{K}$ that is lower in the other cycles. As discussed earlier, this is mainly related to the formation of the SEI during the first cycle. We also observe that when increasing currents are used, the hysteresis between the discharge peak at about $0.3 \mathrm{~V}$ vs $\mathrm{K}^{+} / \mathrm{K}$ and the charge peak at about $0.7 \mathrm{~V}$ vs $\mathrm{K}^{+} / \mathrm{K}$ increases gradually until $1 \mathrm{C}$ and then more abruptly as higher currents (2C and $5 \mathrm{C})$ are used. This change suggests an intrinsic limit of the material due to the slow diffusion of the ions in RP. Moreover, we observed that when a lower current of $\mathrm{C} / 10$ is applied again on the same electrode, an increase of capacity of about $3 \%$ of the ICC occurs. To understand the reason behind this 

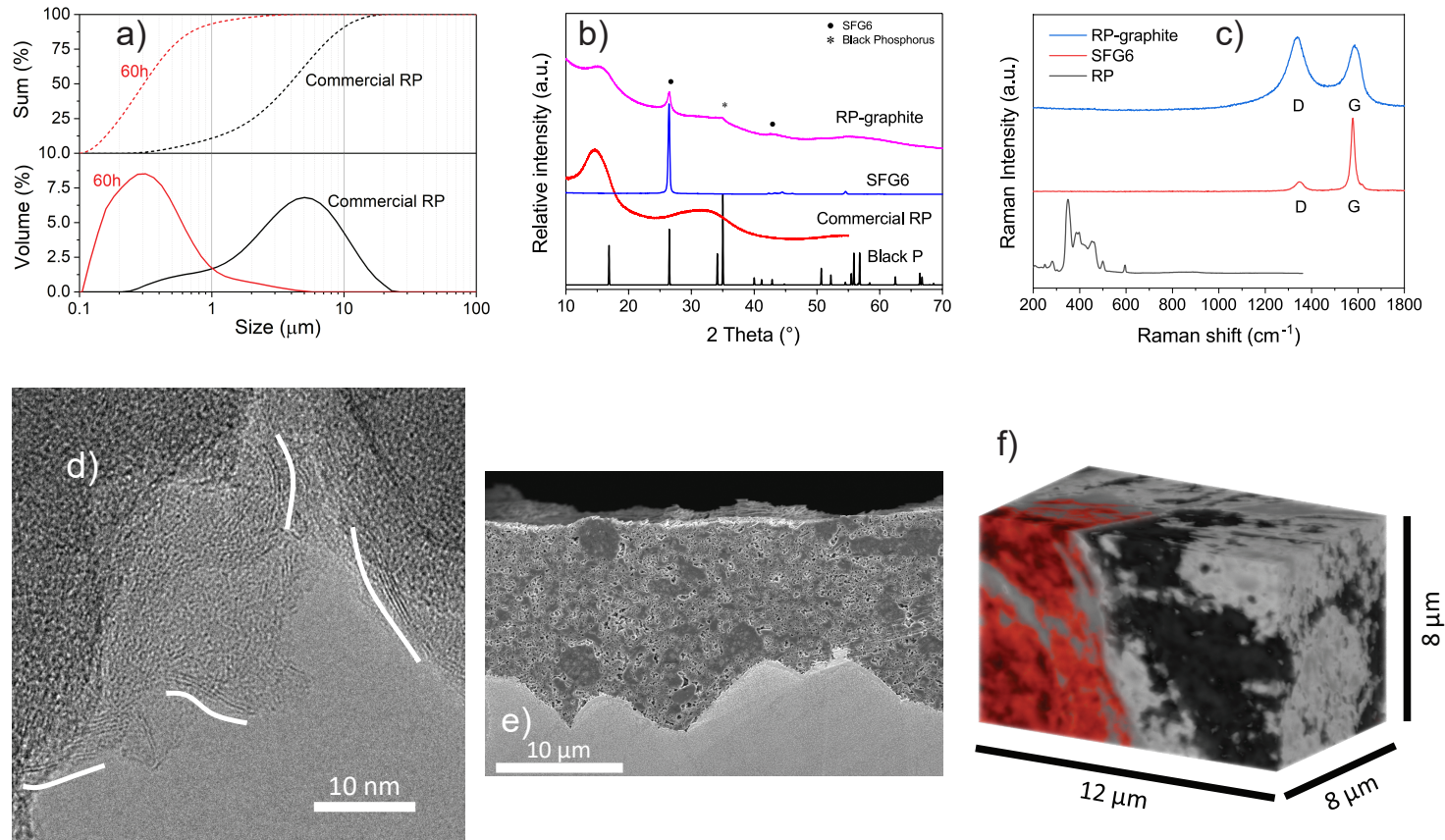

Figure 3. a) XRD analysis of RP, SFG6 and RP-graphite composite. b) Raman spectra of RP, SFG6 and RP-graphite composite. c) Particle size distribution of commercial RP and RP ball milled for 60h. d) TEM of RP-graphite composite. The white lines indicate the presence of graphite layers on the surface of the particles. e) SEM image of the cross section of the electrode and (f) reconstructed section of the PXCT scan of electrode material.

behaviour, we cycled the material at $\mathrm{C} / 20$ for the first three cycles and then at $\mathrm{C} / 10$ for the following cycles while measuring the impedance after every cycle. (Figure S1). We observed that, when a current rate of $\mathrm{C} / 10$ is applied, the capacity increases by about $5 \%$ after 30 cycles $\left(715 \mathrm{mAhg}_{\mathrm{P}}^{-1}\right.$ from $678 \mathrm{mAh}_{\mathrm{P}}^{-1}$, first charge capacity at $\mathrm{C} / 10$ ). (Figure S1a-c) The impedance analysis shows that the charge transfer resistance decreases with the number of cycles (Figure S1d). This can be indicative of an increase of the surface contact between the electrode and the electrolyte, possibly related to the formation of cracks on the electrode caused by the repeated expansion/contraction of RP particles during the alloying reaction, increasing the surface contact. This reduces the path length crossed by the $\mathrm{K}^{+}$ions to reach the bulk of the $\mathrm{RP}$ particles, increasing the capacity. 22 After 100 cycles, the material was still able to hold $95 \%$ of the ICC at C/10 (648.5 $\left.\mathrm{mAh}_{\mathrm{P}}^{-1}\right)$.
To test the material at higher rates, we first cycled it in similar conditions for 40 cycles, then we applied a current rate of $1 \mathrm{C}$ for 100 cycles. A similar increase in capacity was observed for the first 30 cycles at $\mathrm{C} / 10$. At $1 \mathrm{C}$, the material delivered $629 \mathrm{mAh} \mathrm{g}_{\mathrm{P}}^{-1}$ and retained $96.3 \%$ of the capacity after 100 cycles with a coulombic efficiency for each cycle of $99.4 \%$ (Figure 2). The excellent electrochemical properties and cycling stability of this material are likely related to the initial nanosizing of the RP particles and to the homogeneous mixing with a graphite matrix, both contributing to the application of the active material at high currents.

During the synthesis, the particle size distribution, measured using DLS, was greatly reduced after 60 hours of ball milling, resulting in a Dv90 (90\% of the particles have a size lower than this value) of $0.79 \mu \mathrm{m}$ from the original 12.49 (Figure 3 a). This ball milling step did not induce the phase change 

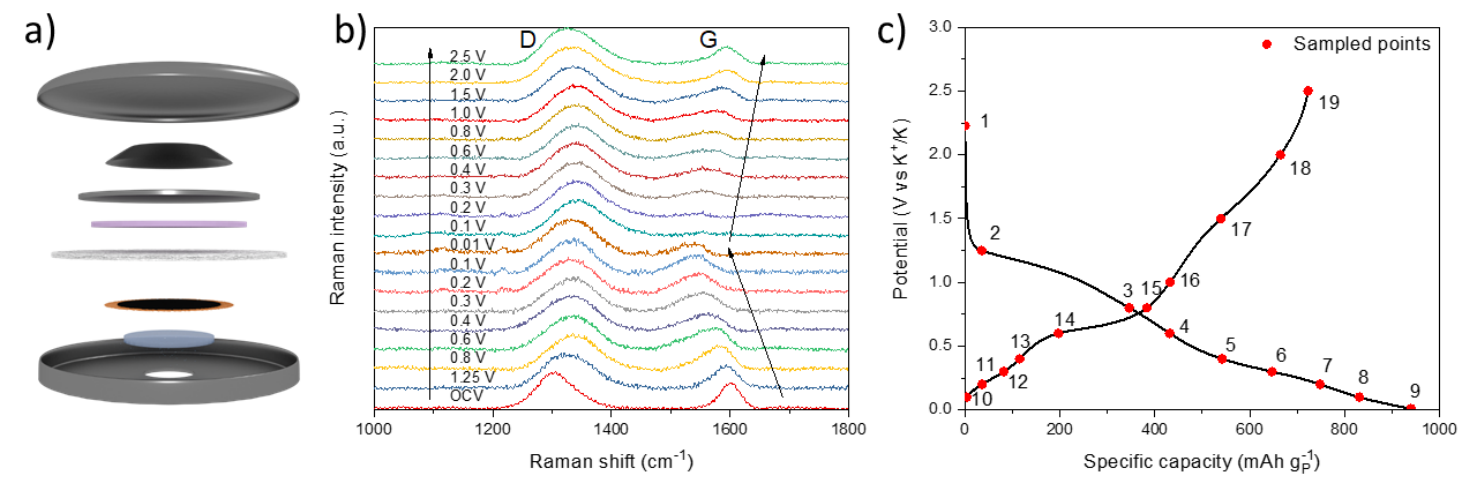

Figure 4. a) Schematic of the cell used for the Raman operando experiment. From top to bottom: cap, spring, spacer, K disk, glass fiber separator, copper grid with electrode material, sapphire window, case. b) Raman spectra acquired on the RP-graphite composite at different states of charge. c) Points on the galvanostatic profile of RP-graphite where the Raman spectra were acquired.

from $\mathrm{RP}$ to the black allotrope (BP), which has been reported to occur when RP is ball milled in dry conditions. 23 The EG that was added during the milling likely reduced the impact energy during milling, as to avoid the phase conversion. BP peaks were not visible in XRD patterns after the ball milling of RP (Figure 3 3 ). Following the size reduction, $\mathrm{RP}$ was mixed for $48 \mathrm{~h}$ with graphite to obtain the RP-graphite composite. XRD patterns showed the presence of a weak peak from $\mathrm{BP} 20$, and broadened graphite peaks, with reduced intensity, suggesting a reduction in particle size and some amorphization (Figure 3b).

The microstructure of the material was investigated with Raman, TEM, SEM and PXCT. Raman spectra acquired of the composite (Figure 3 ;) suggest the carbon has coated the RP particles, as the characteristic peaks of RP in the range 300-500 are not visible $\mathrm{cm}^{-1}, 19,24$ while the $\mathrm{D}$ and $\mathrm{G}$ peak from graphite clearly are visible at $1337 \mathrm{~cm}^{-1}$ and $1586 \mathrm{~cm}^{-1}$ respectively. The broadening of the graphite peaks agree with the XRD that some amorphization of the graphite occurred during the milling, which is a well reported consequence of this procedure. 25 TEM analysis of the composite showed the presence of a few layers of graphite coating the particles, (Figure 3d), supporting the finding in the Raman that a carbon coating was formed during the milling process. This finding moves beyond our previous work which showed the presence of P-C bonds on the outer layers $(16 \mathrm{~nm})$ of RP particles via $\mathrm{x}$-ray photoelectron spectroscopy. 20] In our previous work, we confirmed that the milling time of RP with graphite heavily affects the cyclability of the resulting material, and that long milling times such as $48 \mathrm{~h}$ produce a very stable material with long cycle life with sodium. 20] In this work, we further investigated the morphology of the electrode material by SEM and PXCT analysis. Figure 33,f show a SEM cross section of the electrode and a section of the reconstructed image from the PXCT scan of the electrode material. In (Figure 3f), RP is depicted in red, while graphite is black and Super $\mathrm{P}$ is light grey. The brightness of the colours is indicative of the electron density (dark colour = high electron density). As visible in the PXCT, it is possible to distinguish areas that are more P-rich and C-rich. However the resolution does not allow to clearly distinguish between the particles. SEM coupled with EDX, on the other hand, allow us to better define the composition near the RP particles, showing that the elements are more homogeneously distributed in the material, with some regions where the single elements are more abundant (Figure S2).

To investigate the changes of the material while cycling, we performed an operando Raman experiment. A schematic of the operando cell used is shown in Figure 4a. As explained earlier, only the graphite peaks are visible on the Raman. For this reason, we focused our attention to the carbon peaks during the reaction, in particular on the $G$ peak. This peak has been reported to undergo notable changes during potassiation, due to different stages of intercalation of potassium 
into the graphite layers. 26] As a standard, we performed the experiment on an electrode containing only graphite (Figure S3). We observed that, during potassiation, the peak visible as a shoulder of the $\mathrm{G}$ peak at $1616 \mathrm{~cm}^{-1}$ increases in intensity while the intensity of the $G$ peak decreases. This behaviour has been reported by Chacon-Torres et al., who demonstrated that the relative intensity of the two peaks is indicative of the different potassiation stages of graphite. 27. When graphite is fully potassiated, both peaks disappear and a large asymmetric band appears at about $1550 \mathrm{~cm}^{-1}$. This is related to the interaction between the metallic nature of the potassiated graphite and phonon processes as described by Share et al. 28 The experiment performed on the composite, however, shows a different behaviour. In this case, we observed a constant shift of the $G$ peak to lower Raman shift during potassiation and to higher Raman shift during depotassiation (Figure 4b,c). This finding suggests that this behaviour is not entirely related to the reaction between graphite and potassium (which occurs at $0.25 \mathrm{~V}$ ). Ni et al. described that changes in the position of the $\mathrm{G}$ peak can occur with strain of the graphite layers .29] In particular, they demonstrated that, when the layers are stretched, the position of the $\mathrm{G}$ peak moves to lower Raman shift. We speculate that this phenomenon is what is causing the reversible shift of the $G$ peak in our composite. In our case, the graphite layers that make the carbon coating on the particles can be stretched during the potassiation of $\mathrm{RP}$ and its volume expansion, resulting in about $5 \%$ strain of the layers (calculated from [29]) that could be the cause of the shift of the $G$ peak. As previously mentioned, the shift is reversible, and the $\mathrm{G}$ peak moves to its original position when RP is fully depotassiated.

In Figure 52 and Table S1 the performance of the $\mathrm{RP}-\mathrm{C}$ composite described here are compared to some of the most promising K-ion anodes reported in the literature. For ease of comparison, the capacity is normalised by the total electrode mass (including carbon, additives and binder). The materials selected for comparison are other $\mathrm{P} / \mathrm{C}$ composites prepared by ball milling, carbonaceous anodes such as graphite and hard carbon, and $\mathrm{Sn}_{4} \mathrm{P}_{3}$, with an electrode mass loading in the range of $1.11-1.88 \mathrm{mg} \mathrm{cm}^{-2}$ and a first cycle coulombic efficiency between $50-68 \%$ for P-based electrodes, around $80 \%$ for carbonaceous electrodes and about $66 \%$ for $\mathrm{Sn}_{4} \mathrm{P}_{3}$ based electrode (Figure 5b). Compared to previous works, our material is one of the best performing, especially at high rates. The capacity delivered by the electrode is $403 \mathrm{mAh} \mathrm{g}_{\text {electrode }}^{-1}$ for a current of $24 \mathrm{~mA} \mathrm{~g}_{\text {electrode }}^{-1}$. For a current one hundred times greater, the material is still able to deliver $75.8 \%$ of the capacity, showing its superior performance and capacity retention, similar to graphite and hard carbon. Moreover, the first cycle coulombic efficiency is also very high, second only to carbonaceous materials that, however, deliver a lower capacity. This demonstrating that the nanosizing of RP particles, with a simple and scalable method such as ball milling, prior to mixing with carbon additive is is an effective way to obtain high performing electrodes.

In summary, we synthesized a RP-graphite composite using ball milling to reduce the particle size of RP in a first step and then to mix RP with a decreased particle size with graphite in a second step. The resulting material showed excellent performance with high rate capability up to $5 \mathrm{C}$ and long cycle life at $1 \mathrm{C}$. A maximum capacity of $723 \mathrm{mAhg}^{-1}$ was achieved at $\mathrm{C} / 20$ and $75.8 \%$ of the capacity was retained when currents as high as $5 \mathrm{C}$ was applied. At $1 \mathrm{C}$ the material was able to retain $96.3 \%$ of the capacity after 100 cycles. Analysis on the morphology with SEM-EDX revealed that $\mathrm{P}$ and $\mathrm{C}$ are homogeneously distributed in the electrode. TEM showed that a carbon coating is present on the particles, which could cause the superior performance of the material. Operando Raman analysis showed that the $G$ peak related to the graphite layers reversibly shifts to lower frequency during the RP alloying and moves back to the origin during dealloying. This could be related to the stretching of the graphene layers caused by the volume expansion of the RP particles. This material achieved better performance compared to previous anode materials previously reported, posing as an ideal candidate for application in KIBs.

\section{Experimental section}

\section{Material and composite preparation}

The RP-graphite composite was prepared using a procedure previously reported. 20 $3.4 \mathrm{~g}$ of commercial RP (Sigma Aldrich) was ball milled for $60 \mathrm{~h}$ (FRITSH Pulverisette 7) in a $45 \mathrm{ml}$ zirconia jar with the addition of $10 \mathrm{ml}$ of ethylene glycol (EG) and 180 balls $(\varnothing 5 \mathrm{~mm})$ at $500 \mathrm{rpm}$. The EG serves the purpose to reduce the impact energy of 

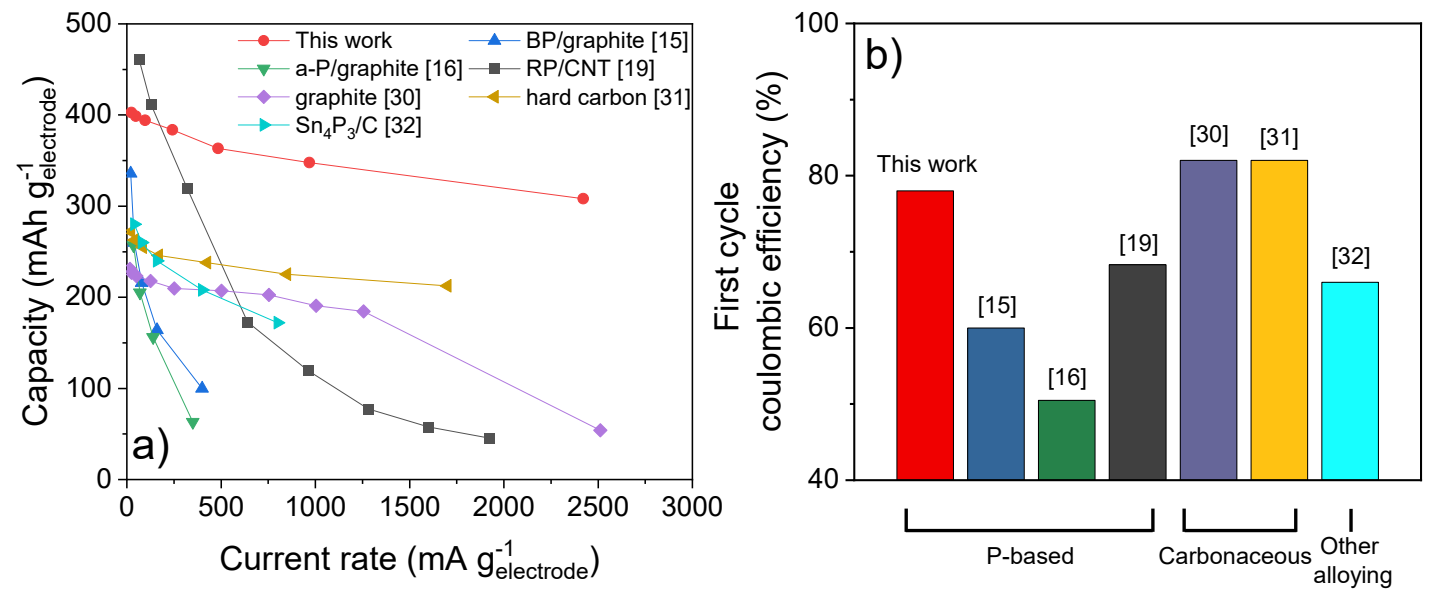

Figure 5. a) Comparison between the performance of the material presented in this work with other anode materials previously reported in literature. b) Comparison of the first cycle coulombic efficiency of the materials. Full details of the electrode materials are shown in table S1. $15,16,19,30,32$

the balls, thus preventing the phase conversion of $\mathrm{RP}$ to black phosphorus. 20, 23 Its high viscosity and low boiling point improves the safety of the procedure by reducing the risk of overpressure. The ball milled RP was then mixed with graphite (SFG6, TIMCAL) (7/3 phosphorus-to-carbon mass ratio) for $48 \mathrm{~h}$ in a PM100 ball mill using a $12 \mathrm{ml}$ stainless steel jar with 5 balls $(\varnothing 1 \mathrm{~cm}$, total mass about $20 \mathrm{~g}$ ) at $500 \mathrm{rpm}$ to ensure a homogeneous mixture of the two components. The resulting RP-graphite composite was mixed with Super P carbon (TIMCAL) and polyacrylic acid binder (PAA, $450 \mathrm{kDa}$, Sigma Aldrich) in a 8:1:1 mass ratio respectively, using NMP as the solvent in a glovebox with low $\mathrm{O}_{2}$ and $\mathrm{H}_{2} \mathrm{O}$ content $(<1 \mathrm{ppm})$. The obtained slurry was cast on a copper foil $35 \mu \mathrm{m}$ thick and let dry overnight in a fume hood. Afterwards, the casting was heated up to $100^{\circ} \mathrm{C}$ under vacuum to remove further residues of solvent. The resulting mass loading was about $1 \mathrm{mg}_{\mathrm{P}} \mathrm{cm}^{2}$ with an average thickness of $15 \mu \mathrm{m}$. The porosity of the casting was visually evaluated via SEM cross sectional analysis and resulted to be about $0.1 .1 \mathrm{~cm} \varnothing$ disks were cut from the casting to be used as electrodes for the electrochemical tests. For the operando Raman experiments, a copper grid was used instead of a foil, to allow the sampling of the electrode material. The casting procedure used is the same as the one previously described. The graphite electrode prepared as standard for the Raman operando was made by mixing pristine SFG6 and PAA binder in a mass ratio 9:1 using NMP as the solvent. The slurry was cast on a copper grid and dried as previously described for the composite.

\section{Physio-chemical characterization}

The RP-graphite composite was characterized using a Rigaku Smartlab 9kW x-ray diffractometer with a $\mathrm{Cu}$ $\mathrm{K} \alpha$ source to interrogate the presence of crystalline impurities. The particle size distribution of RP was measured using dynamic light scattering (DLS) using a Malvern Mastersizer 2000 analyzer. The lasers used for the analysis were a $\mathrm{He} / \mathrm{Ne}(633 \mathrm{~nm})$ and a diode laser $(466 \mathrm{~nm})$. The morphology of the RP and the composite was investigated using a Zeiss Merlin scanning electron microscope with a built-in Oxford Instruments X-Max 150 energy-dispersive Xray (EDX) and a JEOL 3000F field emission gun transmission electron microscope, operated at $300 \mathrm{kV}$. Cross sections of the electrodes were prepared using a a precision etching and coating system II (PECS) Gatan, with an argon ion beam. Operando Raman spectroscopy experiments were performed on a Renishaw InVia Raman microscope using a $512 \mathrm{~nm}$ diode laser. The operando cell used in this experiment is described in the next section. The Raman spectrum of the electrode was acquired 
while the cell was cycling. Ex situ ptychographic X-ray computed tomography (PXCT) was conducted on the I13 beamline at Diamond Light Source in the United Kingdom. The phosphorus anode samples were extracted from the electrode then loaded into a borosilicate glass capillary (diameter of capillary = $100 \mu \mathrm{m})$. The end of capillary was sealed by polymer clay and cyanoacrylate adhesive. The capillary was prepared in an Ar-filled glovebox to prevent air exposure of the sample. A monochromatic beam of $15 \mathrm{keV}$ was selected with a fixed sample-detector distance of $9.5 \mathrm{~m}$, the spatial resolution of each pixel was $55 \mathrm{~nm}$. For each ptychographic tomography scan of phosphorus sample, 900 projections were taken equiangularly from $-90^{\circ}$ to $90^{\circ}$. For each projection, a region of $26 \mu^{2} \times 26 \mu^{2}$ was scanned with a step size of $1.5 \mathrm{\mu m}^{2} \times 1.5 \mathrm{\mu m}^{2}$, with an exposure time of $0.05 \mathrm{~s}$. The obtained projections were processed using the software Blender.

\section{Electrochemical characterization}

To characterize the electrochemical performance of the RP-graphite composite, galvanostatic cycling experiments were performed. The material was tested in a three electrodes setup in a Biologic VMP3, using a EL-cell with $\mathrm{K}$ metal as both the counter and the reference electrode. The experiments were done in triplicate. Potassium bis(fluorosulfonyl)imide (KFSI) 1M in EC:DEC 1:1 was used as the electrolyte. The electrodes were cycled in the potential range $0.01-2.5 \mathrm{~V}$ vs $\mathrm{K}^{+} / \mathrm{K}$ at $\mathrm{C}$ rates ranging from $\mathrm{C} / 20$ to $5 \mathrm{C}$. In this work, we use "discharge" and "charge" to refer to the "potassiation" and "depotassiation" processes, respectively. For the operando Raman measurement, a 2032 coin cell was adapted in order to be used as operando cell. A $4 \mathrm{~mm} \varnothing$ hole was drilled through the center of the positive side of the cell and a $7 \mathrm{~mm}$ $\varnothing$ sapphire window was glued on the inside of the coin cell part using epoxy, to allow the investigation of the electrode material from outside the cell whilst cycling. The cell was assembled using the standard procedure. In the operando experiments, a current rate of $\mathrm{C} / 20$ was applied. Impedance analysis was performed on a three electrode cell with frequencies ranging $200 \mathrm{kHz}-1 \mathrm{~Hz}$, allowing a 1 h rest after every discharge/charge step.

\section{Data availability}

The authors declare that all data supporting the findings of this study are included within the paper and its Supplementary Information files. Source data are available from the corresponding author upon reasonable request.

\section{Acknowledgements}

The authors would like to acknowledge the financial support of the ISCF Faraday Challenge project SOLBAT [grant number FIRG007] and the Henry Royce Institute (through UK Engineering and Physical Science Research Council grant $\mathrm{EP} / \mathrm{R} 010145 / 1)$ for capital equipment. I.C. acknowledges support from a Modentech studentship. We acknowledge Diamond Light Source for time on I13-1 Beamline under Proposal 21960. We are grateful to the David Cockayne Center for Electron Microscopy for the use of their electron microscopes.

\section{Competing Interests}

The authors declare no competing interests.

\section{Author contributions}

I.C and M.P conceived and designed the experiments. I.C. prepared the samples and performed electrochemical tests and Raman, XRD, SEM, EDX and DLS analysis. A.W.X performed the TEM analysis. J.I. wrote the proposal for the PXCT experiment at Diamond Light Source and gave advice for the sample preparation. H.J.L performed the PXCT analysis at Diamond Light Source. J.A contributed to the final manuscript.

\section{Corresponding author}

Prof. Mauro Pasta

Department of Materials, University of Oxford, Parks Road, Oxford OX1 3PH, United Kingdom.

email: mauro.pasta@materials.ox.ac

\section{References}

1. Rechargeable Lithium Batteries (Elsevier, 2015). 
2. Electric Vehicle Outlook 2020 (BloombergNEF, 2020)

3. Mineral Commodity Summaries tech. rep. (2020).

4. Bae, K. Y., Cho, S. H., Kim, B. H., Son, B. D. \& Yoon, W. Y. Energy-Density Improvement in Li-Ion Rechargeable Batteries Based on $\mathrm{LiCoO}_{2}$ $+\mathrm{LiV}_{3} \mathrm{O}_{8}$ and Graphite + Li-Metal Hybrid Electrodes. Materials 12, 2025 (2019).

5. Duan, J. et al. Building Safe Lithium-Ion Batteries for Electric Vehicles: A Review. Electrochemical Energy Reviews 3, 1-42 (2020).

6. Chen, H. et al. Progress in electrical energy storage system: A critical review. Progress in Natural Science 19, 291-312 (2009).

7. Harlow, J. E. et al. A Wide Range of Testing Results on an Excellent Lithium-Ion Cell Chemistry to be used as Benchmarks for New Battery Technologies. Journal of The Electrochemical Society 166, A3031-A3044 (2019).

8. PNNL. Battery500 Consortium to Spark EV Innovations (2016)

9. Deng, T. et al. Layered P2-Type $\mathrm{K}_{0.65} \mathrm{Fe}_{0.5} \mathrm{Mn}_{0.5} \mathrm{O}_{2}$ Microspheres as Superior Cathode for High-Energy Potassium-Ion Batteries. Advanced Functional Materials 28, 1800219 (2018).

10. Kim, H. et al. Investigation of Potassium Storage in Layered P3-Type $\mathrm{K}_{0.5} \mathrm{MnO}_{2}$ Cathode. Advanced Materials 29, 1702480 (2017).

11. Hosaka, T., Kubota, K., Hameed, A. S. \& Komaba, S. Research Development on K-Ion Batteries. Chemical Reviews 120, 6358-6466 (2020).

12. Hurlbutt, K., Wheeler, S., Capone, I. \& Pasta, M. Prussian Blue Analogs as Battery Materials. Joule 2, 1950-1960 (2018).

13. Komaba, S., Hasegawa, T., Dahbi, M. \& Kubota, K. Potassium intercalation into graphite to realize high-voltage/high-power potassium-ion batteries and potassium-ion capacitors. Electrochemistry Communications 60, 172-175 (2015).
14. Carboni, M., Naylor, A. J., Valvo, M. \& Younesi, R. Unlocking high capacities of graphite anodes for potassium-ion batteries. RSC Advances 9, 21070-21074 (2019).

15. Sultana, I., Rahman, M. M., Ramireddy, T., Chen, Y. \& Glushenkov, A. M. High capacity potassium-ion battery anodes based on black phosphorus. Journal of Materials Chemistry A 5, 23506-23512 (2017).

16. Wu, X. et al. Enhanced capacity of chemically bonded phosphorus/carbon composite as an anode material for potassium-ion batteries. Journal of Power Sources 378, 460-467 (2018).

17. Wang, R. et al. Influence of Conductive additives on the stability of red phosphorus-carbon anodes for sodium-ion batteries. Scientific Reports 9, 946 (2019).

18. Capone, I. et al. Electrochemo-Mechanical Properties of Red Phosphorus Anodes in Lithium, Sodium, and Potassium Ion Batteries. Matter 3, 2012-2028 (2020).

19. Chang, W.-C., Wu, J.-H., Chen, K.-T. \& Tuan, H.-Y. Red Phosphorus Potassium-Ion Battery Anodes. Advanced Science 6, 1801354 (2019).

20. Capone, I., Hurlbutt, K., Naylor, A. J., Xiao, A. W. \& Pasta, M. Effect of the Particle-Size Distribution on the Electrochemical Performance of a Red Phosphorus-Carbon Composite Anode for Sodium-Ion Batteries. Energy \& Fuels 33, 4651-4658 (2019).

21. Yang, X. X. et al. Raman spectroscopic determination of the length, strength, compressibility, Debye temperature, elasticity, and force constant of the $\mathrm{C}-\mathrm{C}$ bond in graphene. Nanoscale 4, 502-510 (2012).

22. Ruess, R. et al. Influence of NCM Particle Cracking on Kinetics of Lithium-Ion Batteries with Liquid or Solid Electrolyte. Journal of The Electrochemical Society 167, 100532 (2020).

23. Ramireddy, T. et al. Phosphorus-carbon nanocomposite anodes for lithium-ion and sodium-ion batteries. Journal of Materials Chemistry A 3, 5572-5584 (2015).

24. Durig, J. R. \& Casper, J. M. On the vibrational spectra and structure of red phosphorus. Journal of Molecular Structure 5, 351-358 (1970). 
25. Niwase, K., Tanaka, T., Kakimoto, Y., Ishihara, K. N. \& Shingu, P. H. Raman Spectra of Graphite and Diamond Mechanically Milled with Agate or Stainless Steel Ball-Mill. Materials Transactions, JIM 36, 282-288 (1995).

26. Liu, J. et al. Unraveling the Potassium Storage Mechanism in Graphite Foam. Advanced Energy Materials 9, 1-11 (2019).

27. Chacón-Torres, J. C., Wirtz, L. \& Pichler, T. Raman spectroscopy of graphite intercalation compounds: Charge transfer, strain, and electron-phonon coupling in graphene layers. Physica Status Solidi (B) Basic Research 251, 2337-2355 (2014).

28. Share, K., Cohn, A. P., Carter, R. E. \& Pint, C. L. Mechanism of potassium ion intercalation staging in few layered graphene from in situ Raman spectroscopy. Nanoscale $\mathbf{8}$, 16435-16439 (2016).

29. Ni, Z. H. et al. Uniaxial Strain on Graphene: Raman Spectroscopy Study and Band-Gap Opening. ACS Nano 2, 2301-2305 (2008).

30. Hosaka, T., Kubota, K., Kojima, H. \& Komaba, S. Highly concentrated electrolyte solutions for 4 v class potassium-ion batteries. Chemical Communications 54, 8387-8390 (2018).

31. Yamamoto, H. et al. Synthesizing higher-capacity hard-carbons from cellulose for $\mathrm{Na}$ - and K-ion batteries. Journal of Materials Chemistry A 6, 16844-16848 (2018).

32. Zhang, W., Mao, J., Li, S., Chen, Z. \& Guo, Z. Phosphorus-Based Alloy Materials for Advanced Potassium-Ion Battery Anode. Journal of the American Chemical Society 139, 3316-3319 (2017). 